\title{
CPP-GMR of Co/Cu Multilayered Nanowires Electrodeposited into Anodized Aluminum Oxide Nanochannels with Large Aspect Ratio
}

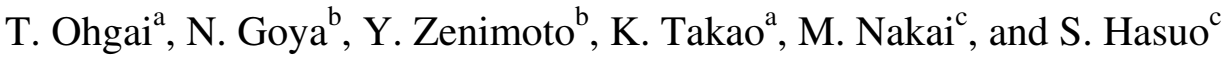 \\ ${ }^{a}$ Division of Chem. \& Mater. Sci., Nagasaki University, Nagasaki 852-8521, JAPAN \\ ${ }^{\mathrm{b}}$ Graduate School of Engineering, Nagasaki University, Nagasaki 852-8521, JAPAN \\ ${ }^{c}$ Kyushu Mitsui Aluminium Co. Ltd., Ohmuta, Fukuoka 836-0067, JAPAN
}

$\mathrm{Co} / \mathrm{Cu}$ multilayered nanowires with alternating $\mathrm{Co}$ and $\mathrm{Cu}$ layers of $10 \mathrm{~nm}$ in thickness were electrodeposited into extremely long nanochannels of anodized aluminium oxide thick films with $60 \mu \mathrm{m}$ in thickness. Growth rate of $\mathrm{Co} / \mathrm{Co}$ multilayered nanowires was around $30 \mathrm{~nm} / \mathrm{sec}$ and the cylindrical shape was precisely transferred from the nanochannels to the nanowires and the aspect ratio reached up to ca. 1,000 to 1 . $\mathrm{Co} / \mathrm{Cu}$ multilayered nanowires with diameter $60 \mathrm{~nm}$ were easily magnetized to the long axis direction of nanowires due to the uni-axial shape anisotropy. $10.5 \%$ of perpendicular giant magnetoresistance effect was observed in $\mathrm{Co} / \mathrm{Cu}$ multilayered nanowires with $3,000 \mathrm{Co} / \mathrm{Cu}$ bilayers.

\section{Introduction}

An array of nanowires with a large aspect ratio has received much attention due to the nanowires' unique shape anisotropy and extremely large surface area. Morales et al reported that semiconductor nanowires were synthesized using a laser ablation cluster formation technique and a vapor-liquid-solid (VLS) growth technique (1). They obtained Si nanowires with diameters of 6 to $20 \mathrm{~nm}$ and Ge nanowires with diameters of 3 to $9 \mathrm{~nm}$. As a biological application of nanowires, Kim et al reported that a ferromagnetic Ni-Si alloy nanowires array was synthesized using Ag-assisted wet chemical etching technique (2). The Ni-Si alloy nanowires with diameter of 60 100 nm and length of 5 10 $\mu \mathrm{m}$ were obtained and T-cell separation was demonstrated using the extremely large specific surface area of the nanowires array. Nanowires can be fabricated by stretching a microsize wire down to nano-size or manipulating atoms using a scanning probe under vacuum condition, while they can be also prepared by electrodepositing metallic atoms into a nanochannel (3).

Membrane thin films with numerous nanochannels can be used as a template electrode for electrodeposition of nanowires (4). For example, ion-track etched polycarbonate membrane filters and anodized aluminum oxide membrane filters are known to be typical nanochannel template for growing nanowires (5). The array of magnetic nanowires synthesized into the nanochannel can be applied to a perpendicular magnetic storage media with high density domain or a high sensitive magnetic field sensor (6). Whitney et al reported that the arrays of $\mathrm{Ni}$ and $\mathrm{Co}$ nanowires were electrodeposited in polymer templates with the nanometer-sized pores prepared by nuclear track etching technique (7). They found that the preferred magnetization direction 
is perpendicular to the film plane and a coercive force of as high as 680 Oe was reached. Piraux et al reported that the array of $\mathrm{Co} / \mathrm{Cu}$ multilayered nanowires with giant magnetoresistance (GMR) response was electrodeposited in nanoporous polymer template (8). In the report, magnetoresistance measurements with the current perpendicular to the layers were performed on the array of parallel nanowires. They observed that the $15 \%$ of GMR at room temperature on $\mathrm{Co} / \mathrm{Cu}$ multilayered nanowires with length of several $\mu \mathrm{m}$. However, for the micro-electronic device application, it is required to control precisely the diameter and length of nanowires with a wide range of aspect ratios. In this study, to synthesize a novel GMR sensor, $\mathrm{Co} / \mathrm{Cu}$ multilayered nanowires were electrodeposited into an anodized aluminum oxide template with extremely large aspect ratio more than 1,000 to 1 .

\section{Experimental}

Figure 1 shows the experimental apparatus and time-dependence of the applied voltage to synthesize an anodized aluminum oxide membrane filter. Anodized aluminum oxide thick films with numerous nanochannels were exfoliated mechanically by the pressure of hydrogen gas generated at the interface between an oxide layer and a metallic aluminum during the subsequent cathodic reduction process after the growing of anodic aluminum oxide layer. First, an anodization voltage of $70 \mathrm{~V}$ was applied to growing aluminum oxide nanochannel. Next, the anodization voltage was decreased gradually down to $0 \mathrm{~V}$ for thinning the pore bottom oxide layer (barrier layer). Finally, cathodic voltage was applied to exfoliate an anodized aluminum oxide membrane film from the metallic aluminum rod due to the hydrogen evolution. These anodized aluminum oxide films were used as a template for growing nanowires.

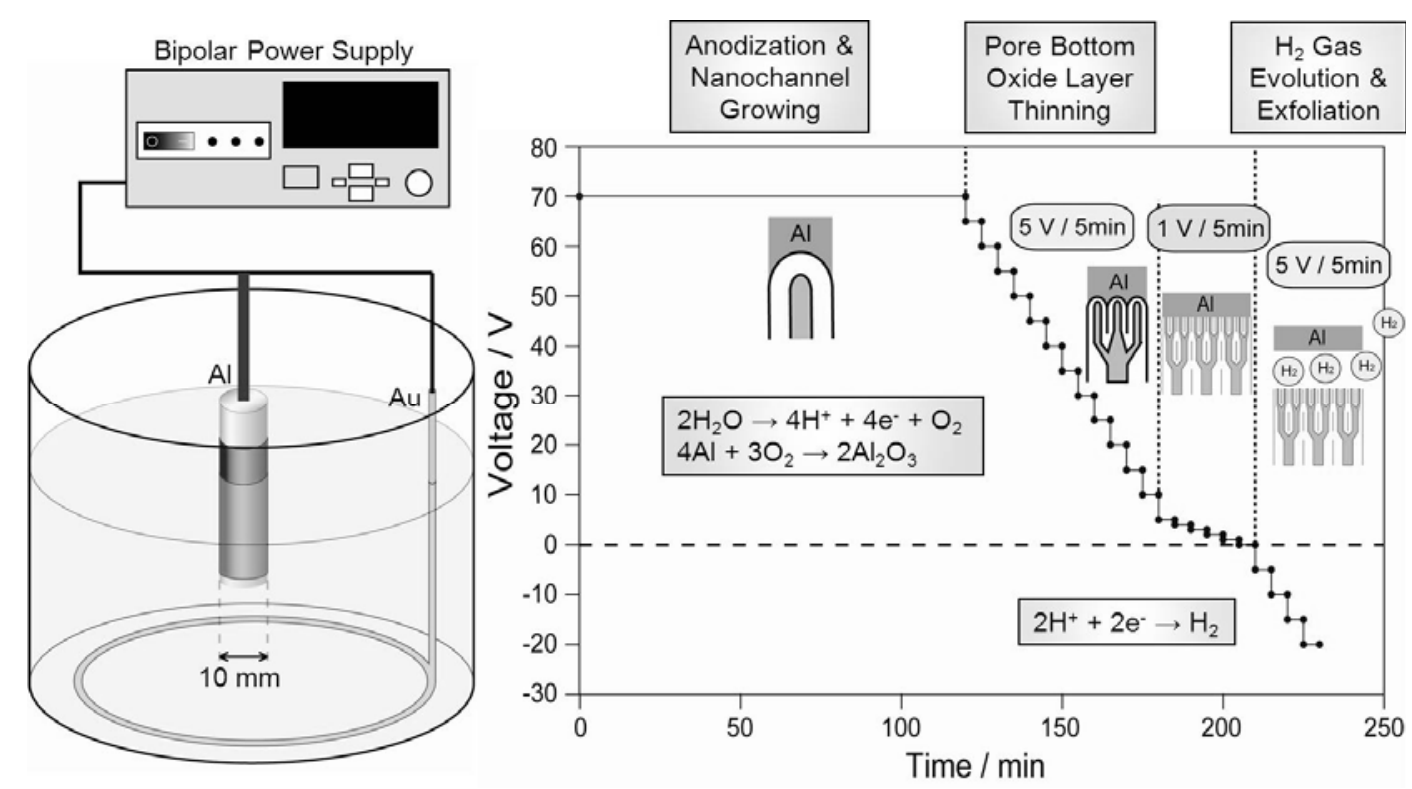

Figure 1. Experimental apparatus (left) and time-dependence of applied voltage (right) to synthesize an anodized aluminum oxide membrane filter.

Figure 2 shows the experimental apparatus and a nanochannel template for electrodeposition of $\mathrm{Co} / \mathrm{Cu}$ multilayered nanowires. On the surface of the membrane filter, a gold layer was sputter-deposited to cover the pores and make a cathode. A gold 
wire and $\mathrm{Ag} / \mathrm{AgCl}$ electrode were used as an anode and a reference electrode. An aqueous solution containing $\mathrm{CoSO}_{4}(120 \mathrm{~g} / \mathrm{L}), \mathrm{CuSO}_{4}(1.6 \mathrm{~g} / \mathrm{L})$ and $\mathrm{H}_{3} \mathrm{BO}_{3}(45 \mathrm{~g} / \mathrm{L})$ was used as electrolyte. $\mathrm{Co} / \mathrm{Cu}$ multilayered nanowires with alternating $\mathrm{Co}$ and $\mathrm{Cu}$ layers of $10 \mathrm{~nm}$ in thickness were electrodeposited into extremely long nanochannels of anodized aluminum oxide films with $60 \mu \mathrm{m}$ in thickness.
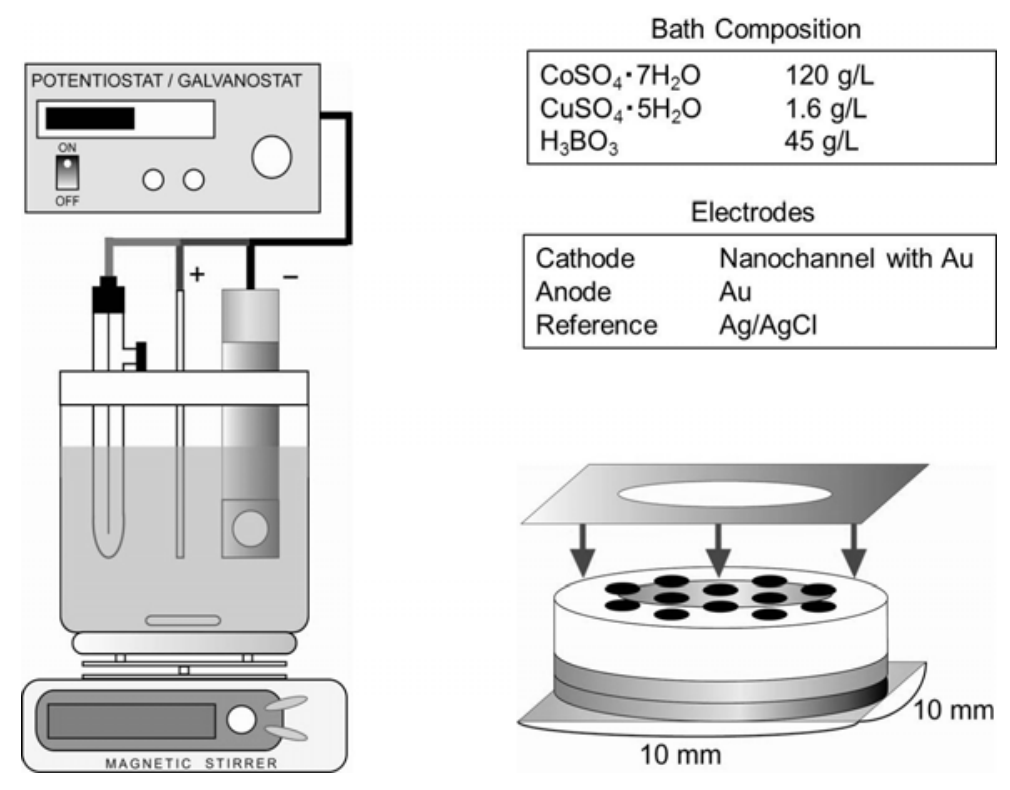

Figure 2. Experimental apparatus (left), electroplating information (top, right) and a nanochannel template (bottom, right) for electrodeposition of $\mathrm{Co} / \mathrm{Cu}$ multilayered nanowires.

After growing the nanowires, the anodized aluminum oxide films were dissolved in an aqueous solution containing sodium hydroxide and the remains consisted of nanowires and a gold layer which served as a sample for SEM observation. Magnetic hysteresis loops and magnetoresistive curves of the nanowires were obtained using Vibrating Sample Magnetometer (VSM) and LCR meter with increasing the magnetic field up to 10 kOe. Magnetic field was applied to in-plane and perpendicular directions against to the membrane film plane as shown in Figure 3. This perpendicular direction corresponds to the parallel direction to the long axis of nanowires.

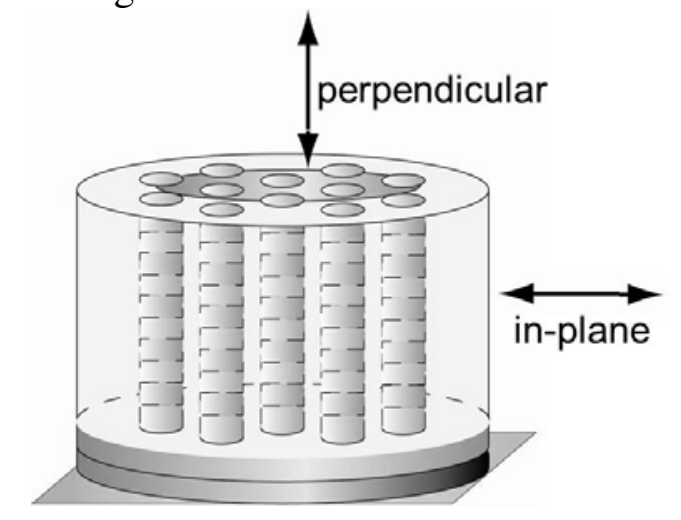

Figure 3. Magnetic field directions for measuring the magnetization and magnetoresistance of $\mathrm{Co} / \mathrm{Cu}$ multilayered nanowires. 


\section{Results and Discussion}

\section{Anodization Process of Aluminum}

Figure 4 shows an optical micrograph of the anodized aluminum oxide membrane, as well as an SEM picture of the nanochannels exfoliated from a metallic aluminum rod. Round and disc shaped membrane filters with diameters of $10 \mathrm{~mm}$ were obtained as shown in Figure 4. The average channel diameter was around $60 \mathrm{~nm}$ and the channel density was around $10^{8}$ channel $/ \mathrm{cm}^{2}$ while the channel length was ca. $60 \mu \mathrm{m}$.
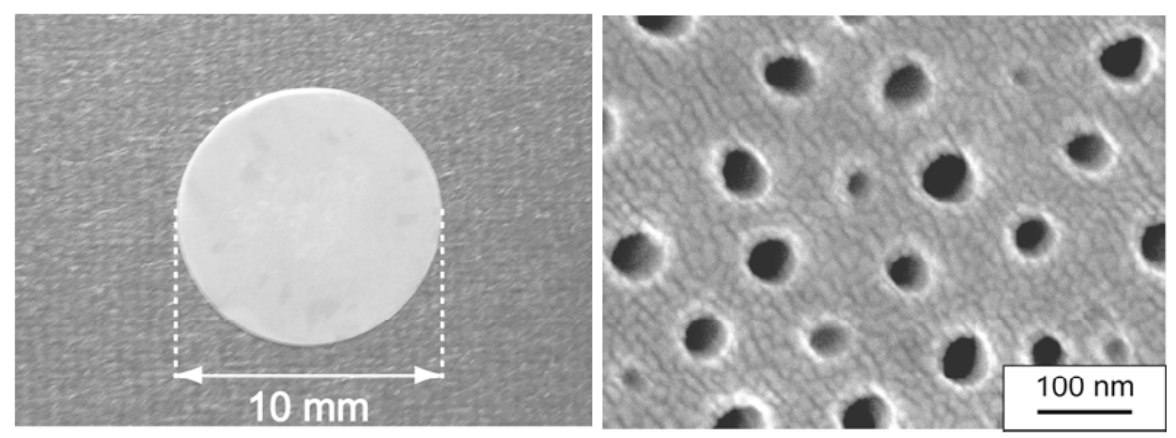

Figure 4. An optical micrograph of the anodized aluminum oxide membrane (left), as well as an SEM picture of the nanochannels (right) exfoliated from an metallic aluminum rod.

\section{Electrodeposition Process of $\mathrm{Co} / \mathrm{Cu}$ Multilayered Nanowires}

Figure 5 shows the time-dependence of the cathodic current during electrodeposition of $\mathrm{Co} / \mathrm{Cu}$ multilayered nanowires. When the nanowires reach to the membrane surface, the current will increase drastically due to the formation of hemispherical deposits. Therefore, the growth rates can be estimated from dividing channel length by the filling time. As shown in Figure 5, the filling time was around 2,000 s and the deposition rate is estimated to be about $30 \mathrm{~nm} / \mathrm{s}$.

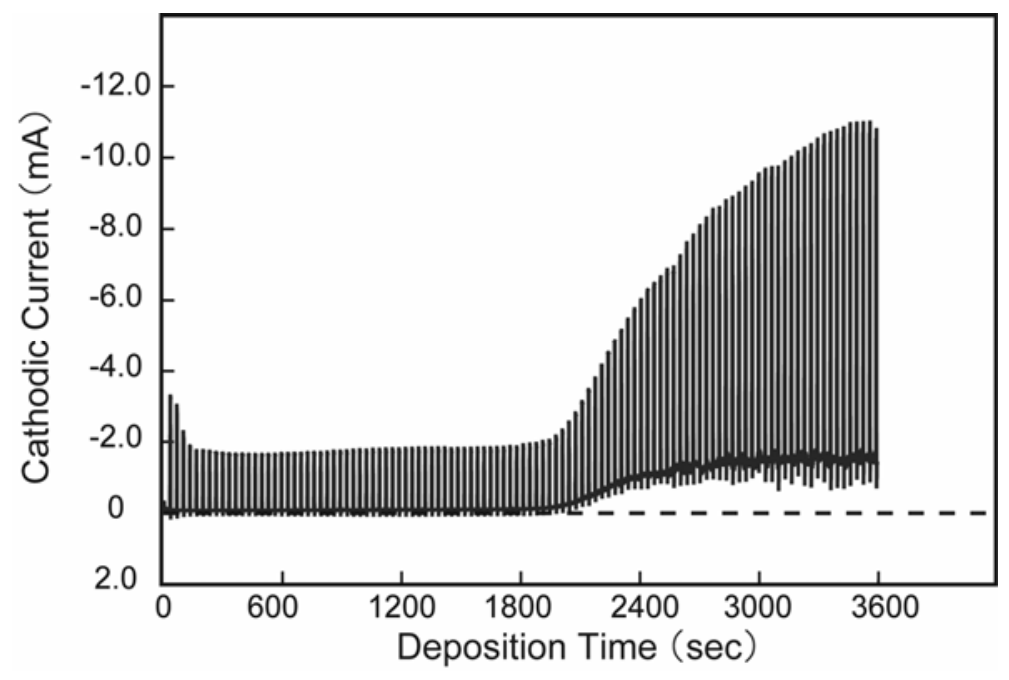

Figure 5. Time-dependence of the cathodic current during electrodeposition of $\mathrm{Co} / \mathrm{Cu}$ multilayered nanowires. 


\section{$\underline{\text { Magnetization and Magnetoresistance of } \mathrm{Co} / \mathrm{Cu} \text { Multilayered Nanowires }}$}

Figure 6 shows magnetic hysteresis loops of electrodeposited $\mathrm{Co} / \mathrm{Cu}$ multilayered nanowires with a diameter of $60 \mathrm{~nm}$ and a length of $60 \mu \mathrm{m}$. Typical perpendicular magnetization behavior was observed and the coercive force of $\mathrm{Co} / \mathrm{Cu}$ multilayered nanowires was ca. $1 \mathrm{kOe}$. This is resulting from the shape anisotropy of nanowires with very large aspect ratio of approx. 1,000 to 1. Figure 7 shows magnetoresistive hysteresis of electrodeposited $\mathrm{Co} / \mathrm{Cu}$ multilayered nanowires with diameter of $60 \mathrm{~nm}$ and length of $60 \mu \mathrm{m}$. The resistance of $\mathrm{Co} / \mathrm{Cu}$ multilayered nanowires was decreased with increase in magnetic field and $10.5 \%$ of giant magnetoresistance effect was observed in the multilayered nanowires with Co layer $10 \mathrm{~nm}, \mathrm{Cu}$ layer $10 \mathrm{~nm}$ and 3,000 bi-layers.

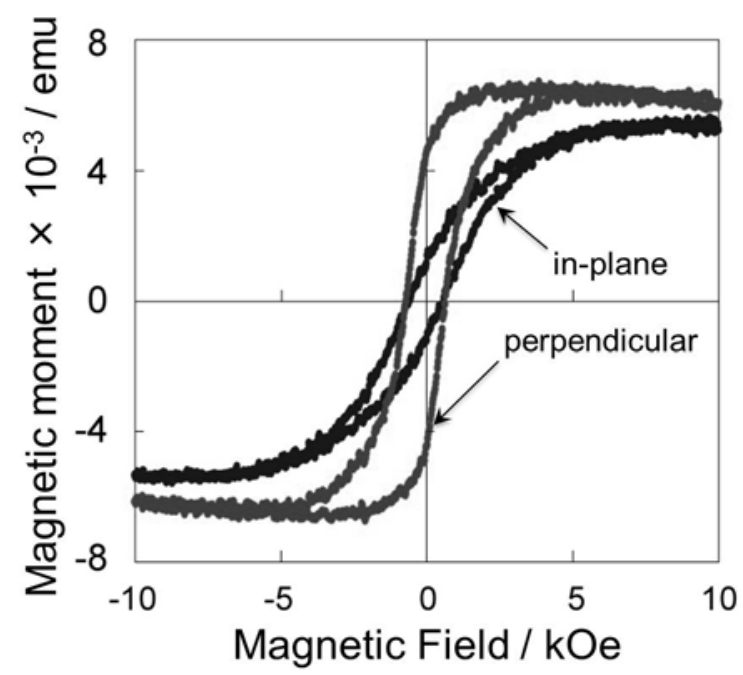

Figure 6. Magnetic hysteresis loops of electrodeposited $\mathrm{Co} / \mathrm{Cu}$ multilayered nanowires with a diameter of $60 \mathrm{~nm}$ and a length of $60 \mu \mathrm{m}$.

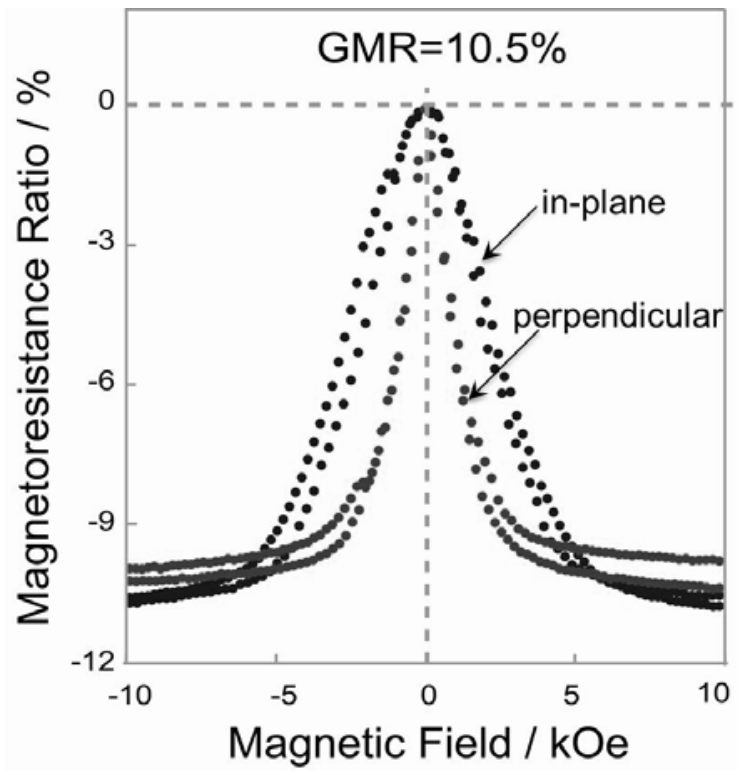

Figure 7. Magnetoresistive hysteresis of electrodeposited $\mathrm{Co} / \mathrm{Cu}$ multilayered nanowires with diameter of $60 \mathrm{~nm}$ and length of $60 \mu \mathrm{m}$. 


\section{Conclusions}

Anodized aluminum oxide membrane filters containing channels with $60 \mathrm{~nm}$ diameter and $60 \mu \mathrm{m}$ length were synthesized using a bipolar continuous electrolysis process with anodic oxidation and cathodic exfoliation. Co/Cu multilayered nanowires with $\mathrm{Co}-10 \mathrm{~nm}$, $\mathrm{Cu}-10 \mathrm{~nm}$ and 3,000 bi-layers were synthesized using a pulsed current electrodeposition technique. A giant magnetoresistance effect of $10.5 \%$ was observed in $\mathrm{Co} / \mathrm{Cu}$ multilayered nanowires electrodeposited into anodized aluminum oxide template with super large aspect ratio of ca. 1,000.

\section{Acknowledgments}

This work was supported in part by Kyushu Industrial Technology Center, Mitutoyo Association for Science \& Technology, Yazaki Memorial Foundation for Science \& Technology and Research Foundation for Materials Science.

\section{References}

1. A.M. Morales and C.M. Lieber, Science 279, 208 (1998).

2. D.J. Kim, J.K. Seol, M.R. Lee, J.H. Hyung, G.S. Kim, T. Ohgai and S.K. Lee, Appl. Phys. Lett., 100, 163703 (2012).

3. C.R. Martin, Adv. Mater. 3, 457 (1991).

4. T. Ohgai, K. Hjort, R. Spohr and R. Neumann, J. Appl. Electrochem., 38, 713 (2008).

5. R. Spohr, C. Zet, B.E. Fischer, H. Kiesewetter, P. Apel, I. Gunko, T. Ohgai and L. Westerberg, Nucl. Inst. Meth. Phys. Res. B, 268, 676 (2010).

6. T. Ohgai, I. Enculescu, C. Zet, L. Westerberg, K. Hjort, R. Spohr and R. Neumann, J. Appl. Electrochem., 36, 1157 (2006).

7. T.M. Whitney, J.S. Jiang, P.C. Searson and C.L. Chien, Science 261, 1316 (1993).

8. L. Piraux, J.M. George, J.F. Despres, C. Leroy, E. Ferain, R. Legras, K. Ounadjela and A. Fert, Appl. Phys. Lett., 65, 2484 (1994). 